\title{
UNIQUENESS OF SOLUTION TO SYSTEMS OF ELLIPTIC OPERATORS AND APPLICATION TO ASYMPTOTIC SYNCHRONIZATION OF LINEAR DISSIPATIVE SYSTEMS
}

\author{
TAtsien Li ${ }^{1}$ AND Bopeng RAO ${ }^{2,3, *}$
}

\begin{abstract}
We show that under Kalman's rank condition on the coupling matrices, the uniqueness of solution to a complex system of elliptic operators can be reduced to the observability of a scalar problem. Based on this result, we establish the asymptotic stability and the asymptotic synchronization for a large class of linear dissipative systems.
\end{abstract}

Mathematics Subject Classification. 93B05, 93C20, 35L53.

Received July 3, 2020. Accepted September 22, 2020.

\section{INTRODUCTION AND MAIN RESULTS}

Let $H$ and $V$ be two separated Hilbert spaces such that $V \subset H$ with dense and compact imbedding. Let $L$ be the duality mapping from $V$ onto its dual $V^{\prime}$, and $\gamma$ be a linear continuous operator from $V$ into $V^{\prime}$.

Denote by $\mathcal{V}$ and $\mathcal{H}$ the product spaces:

$$
\mathcal{V}=V^{N}, \quad \mathcal{H}=H^{N}
$$

Let $U=\left(u^{(1)}, \ldots, u^{(N)}\right)^{T}$. Define the vector operators $\mathcal{L}$, respectively, $\mathcal{G}$ by

$$
\mathcal{L} U=\left(\begin{array}{c}
L u^{(1)} \\
\vdots \\
L u^{(N)}
\end{array}\right), \quad \mathcal{G} U=\left(\begin{array}{c}
\gamma u^{(1)} \\
\vdots \\
\gamma u^{(N)}
\end{array}\right)
$$

Let $A$ and $D$ be symmetric and positive semi-definite matrices. Consider the following second order evolution system

$$
U^{\prime \prime}+\mathcal{L} U+A U+D \mathcal{G} U^{\prime}=0
$$

Keywords and phrases: uniqueness, elliptic systems, asymptotic synchronization, condition of compatibility, Kalman's rank condition.

1 Shanghai Key Laboratory for Contemporary Applied Mathematics, Nonlinear Mathematical Modeling and Methods Laboratory, School of Mathematical Sciences, Fudan University, Shanghai 200433, P.R. China.

2 Institut de Recherche Mathématique Avancée, Université de Strasbourg, 67084 Strasbourg, France.

3 School of Mathematical Sciences, Qufu Normal University, Qufu 273165, P.R. China.

* Corresponding author: rao@math.unistra.fr 
where " I" denotes the time derivative.

Clearly, system (1.3) generates a semi-group of contractions in the space $\mathcal{V} \times \mathcal{H}$. Moreover, according to Foias' theory of spectral decomposition (see Thm. 3.4), the asymptotic stability of the dissipative system (1.3) is equivalent to the uniqueness of solution to the following over-determined system for the variable $\Phi=\left(\phi^{(1)}, \cdots, \phi^{(N)}\right)^{T}$ :

$$
\mathcal{L} \Phi+A \Phi=\beta^{2} \Phi
$$

associated with the $D$-observation

$$
D \mathcal{G} \Phi=0 .
$$

We first observe that Kalman's rank condition

$$
\operatorname{rank}\left(D, A D, \ldots, A^{N-1} D\right)=N
$$

is necessary for the uniqueness of solution to the over-determined system (1.4)-(1.5), therefore, necessary for the asymptotic stability of system (1.3) (see Props. 2.2 and 3.3). But, it is not sufficient in general as shown by an example in [16].

The first objective of the present work is to find an efficient characterization for the uniqueness of solution to the over-determined system (1.4)-(1.5). Since the matrix $D$ in (1.6) is not invertible in general, the $D$-observation (1.5) cannot imply the nullity of the full observation

$$
\gamma \phi^{(i)}=0, \quad i=1, \cdots, N .
$$

So, the following theorem is different from Carleman's classic unique continuation theorem on elliptic operators in [29], [5] or [10].

Theorem 1.1. Let the pair $(A, D)$ satisfy Kalman's rank condition (1.6). Assume that there exists a real number a such that $\|A-a I\|$ is small enough. Assume furthermore that there exists $c>0$ independent of $\beta$ and $f$, such that any solution $\phi \in V$ to the following over-determined scalar problem

$$
\beta^{2} \phi-L \phi=f \quad \text { and } \quad \gamma \phi=0
$$

satisfies the uniform estimation:

$$
\|\phi\|_{H} \leqslant c\|f\|_{H}
$$

Then, the over-determined system (1.4)-(1.5) has only the trivial solution $\Phi \equiv 0$.

Thus, "under Kalman's rank condition, the observability of a scalar problem implies the uniqueness of solution to a complex system." Using this method, we may provide a direct and efficient approach to solve a seemingly difficult problem of uniqueness of a complex system.

When the matrices $A$ and $D$ do not satisfy Kalman's rank condition (1.6), the uniqueness theorem fails and system (1.3) is not asymptotically stable. We return to consider the asymptotic synchronization by $p$-groups, a weakened notion of stability, described as follows.

Let $p \geqslant 1$ be an integer and

$$
0=n_{0}<n_{1}<n_{2}<\cdots<n_{p}=N
$$


be integers such that $n_{r}-n_{r-1} \geqslant 2$ for $1 \leqslant r \leqslant p$. We re-arrange the components of the state variable $U$ into $p$ groups

$$
\left(u^{(1)}, \cdots, u^{\left(n_{1}\right)}\right),\left(u^{\left(n_{1}+1\right)}, \cdots, u^{\left(n_{2}\right)}\right), \cdots \cdots,\left(u^{\left(n_{p-1}+1\right)}, \cdots, u^{\left(n_{p}\right)}\right) .
$$

Definition 1.2. System (1.3) is asymptotically synchronizable by $p$-groups if for any given initial data $\left(U_{0}, U_{1}\right) \in$ $\mathcal{V} \times \mathcal{H}$, the corresponding solution $U$ to system (1.3) satisfies

$$
\left(u^{(k)}(t)-u^{(l)}(t),\left(u^{(k)}\right)^{\prime}(t)-\left(u^{(l)}\right)^{\prime}(t)\right) \rightarrow(0,0) \quad \text { in } V \times H
$$

as $t \rightarrow+\infty$ for all $n_{r-1}+1 \leqslant k, l \leqslant n_{r}$ and $1 \leqslant r \leqslant p$.

The exact boundary synchronization and the approximate boundary synchronization on a finite interval for a coupled system of wave equations with various boundary conditions were studied in the monograph [20].

The above asymptotic synchronization investigates the behavior of solutions on the infinite horizon. So, the notion of synchronizable state is no longer available as in the case of approximate boundary synchronization at a finite horizon (see $[16,17,19]$ ). This certainly raises interesting questions and needs new effective methods.

Now let us outline the main idea in the study of asymptotic synchronization.

Theorem 1.3. Assume that system (1.3) is asymptotically synchronizable by p-groups. Then, we have the following rank condition

$$
\operatorname{rank}\left(D, A D, \cdots, A^{N-1} D\right) \geqslant N-p .
$$

Moreover, we have

Theorem 1.4. Assume that system (1.3) is asymptotically synchronizable by p-groups under the minimal rank condition:

$$
\operatorname{rank}\left(D, A D, \cdots, A^{N-1} D\right)=N-p .
$$

Then there exists $\alpha_{r s}$ such that the matrix $A=\left(a_{i j}\right)$, respectively, the matrix $D=\left(d_{i j}\right)$ satisfies the following row-sum conditions by blocks

$$
\sum_{j=n_{s-1}+1}^{n_{s}} a_{i j}=\alpha_{r s}, \quad \text { respectively, } \sum_{j=n_{s-1}+1}^{n_{s}} d_{i j}=0
$$

for all $n_{r-1}+1 \leqslant i \leqslant n_{r}$ and $1 \leqslant r, s \leqslant p$.

The above conditions will be called the conditions of $C_{p}$-compatibility, which are important ingredients in the study of asymptotic synchronization. In Proposition 4.6, we will show that these conditions guarantee the existence of symmetric and positive semi-definite matrices $\bar{A}_{p}$ and $\bar{D}_{p}$ of order $(N-p)$, such that the asymptotic synchronization by $p$-groups of system (1.3) is equivalent to the asymptotic stability of the following reduced system with an auxiliary variable $W=\left(w^{(1)}, \cdots, w^{(N-p)}\right)^{T}$ :

$$
W^{\prime \prime}+\mathcal{L} W+\bar{A}_{p} W+\bar{D}_{p} G W^{\prime}=0 .
$$

The above approach is direct and efficient. However, the necessity of the conditions of compatibility is a delicate question. In fact, these conditions are usually imposed as a physically reasonable hypotheses for systems of ordinary differential equations. Theorem 1.4 clarifies that the necessity of these conditions of compatibility is 
in fact the consequence of the minimality of the rank of Kalman's matrix. This makes the theory of asymptotic synchronization more complete for systems of partial differential equations.

Moreover, since the reduced matrices $\bar{A}_{p}$ and $\bar{D}_{p}$ are symmetric and positive semi-definite, the asymptotic stability of system (1.16) is equivalent to the uniqueness of the following over-determined system for the reduced variable $\Psi=\left(\psi^{(1)}, \cdots, \psi^{(N-p)}\right)^{T}$ :

$$
\mathcal{L} \Psi+\bar{A}_{p} \Psi=\beta^{2} \Psi
$$

associated with the $\bar{D}_{p}$-observation

$$
\bar{D}_{p} \mathcal{G} \Psi=0 .
$$

The paper is organized as follows. Section 2 is devoted to the uniqueness theorem. Then, in Section 3, we apply this theorem to an abstract linear evolution equation and propose an easier way to get the asymptotic stability. Section 4 is devoted to the study of the asymptotic synchronization by $p$-groups. We first give a lower bound on the rank of Kalman's matrix, which is necessary for the asymptotic synchronization by $p$-groups, then we show that the necessity of the conditions of $C_{p}$-compatibility is in fact a consequence of the minimality of the rank of Kalman's matrix (Thms. 4.7 and 4.10). In Section 5, we give some applications to the system of wave equations with boundary damping or locally distributed damping. Moreover, we investigate a system of Kirchhoff plate equations with boundary shear force damping. In Section 6, we raise some possible enlargements on the topic.

The main results of this work were announced in [21].

\section{UNIQUENESS THEOREM}

We will reformulate Theorem 1.1 for a general matrix $A$ of order $N$ and a general matrix $D$ of order $N \times M$. In particular, the matrix $A$ is not necessarily of "cascade type" as in the literature.

Let $H$ and $V$ be two separated Hilbert spaces such that $V \subset H$ with dense and compact imbedding. Let $L$ be the duality mapping from $V$ onto its dual space $V^{\prime}$, such that

$$
\langle L \phi, \psi\rangle_{V^{\prime}, V}=(\phi, \psi)_{V}, \quad \forall \phi, \psi \in V .
$$

Let $\gamma$ be a linear continuous operator from $V$ into $V^{\prime}$, such that

$$
\langle\gamma \phi, \psi\rangle_{V^{\prime}, V}=\langle\gamma \psi, \phi\rangle_{V^{\prime}, V}
$$

and

$$
\langle\gamma \phi, \phi\rangle_{V^{\prime}, V} \geqslant 0 \text { and }\langle\gamma \phi, \phi\rangle_{V^{\prime}, V}=0 \text { if and only if } \gamma \phi=0 .
$$

Recall that $\mathcal{L}$ and $\mathcal{G}$ are defined by (1.2). Consider the eigen-system for the variable $\Phi=\left(\phi^{(1)}, \cdots, \phi^{(N)}\right)^{T}$ :

$$
\mathcal{L} \Phi+A^{T} \Phi=\beta^{2} \Phi
$$

associated with the $D$-observation

$$
D^{T} \mathcal{G} \Phi=0
$$

Let us recall the following generalized rank condition of Kalman's type, which will play an important role in the study of uniqueness. 
Proposition 2.1. (Lem. 2.1 in [16]) Let $d \geqslant 0$ be an integer. The rank condition

$$
\operatorname{rank}\left(D, A D, \cdots, A^{N-1} D\right)=N-d
$$

holds if and only if $d$ is the largest dimension of the subspaces which are invariant for $A^{T}$ and contained in $\operatorname{Ker}\left(D^{T}\right)$.

Proposition 2.2. Assume that system (2.4)-(2.5) has only the trivial solution. Then the pair $(A, D)$ necessarily satisfies Kalman's rank condition (1.6).

Proof. If (1.6) fails, by Proposition 2.1, there exist a number $\lambda \in \mathbb{R}$ and a non-trivial vector $E \in \mathbb{R}^{N}$, such that

$$
A^{T} E=\lambda E \quad \text { and } \quad D^{T} E=0 .
$$

Noting that $L$ is self-adjoint and the imbedding from $V$ into $H$ is compact, by the spectral theory of compact self-adjoint operators, there exist $v \in V$ and $\alpha \in \mathbb{R}^{+}$large enough, such that $L v=\alpha v$. Defining

$$
\beta^{2}=\alpha+\lambda>0 \text { and } \Phi=v E
$$

it is easy to check that $\Phi$ is a solution to (2.4). Moreover, noting that $G$ is of diagonal form, we have

$$
D^{T} \mathcal{G} \Phi=\gamma v D^{T} E=0 .
$$

Thus, we get a non-trivial solution of (2.4)-(2.5), which contradicts the assumption.

Definition 2.3. Let $\lambda_{1}, \cdots, \lambda_{m}$ denote the distinct eigenvalues of $A$. The matrix $A$ satisfies the $\epsilon$-closing condition if there exists a number $a$ such that

$$
\sup _{1 \leqslant l \leqslant m}\left|\lambda_{l}-a\right| \leqslant \epsilon
$$

Definition 2.4. The operator $L$ satisfies the $c$-gap condition if there exists a number $c>0$, such that

$$
\left|\alpha_{n}-\alpha_{n^{\prime}}\right| \geqslant c
$$

holds true for all distinct eigenvalues $\alpha_{n} \neq \alpha_{n^{\prime}}$ of $L$.

Definition 2.5. The pair $(L, \gamma)$ is observable if there exists a constant $c>0$, independent of $\beta \in \mathbb{R}$ and $f \in H$, such that the observability inequality

$$
c\|\phi\|_{H} \leqslant\|f\|_{H}
$$

holds for any given solution $\phi \in V$ to the following over-determined scalar problem

$$
\beta^{2} \phi-L \phi=f \quad \text { with } \quad \gamma \phi=0 .
$$

Theorem 2.6. Assume that conditions (2.1)-(2.3) hold true. Assume furthermore that the pair $(A, D)$ satisfies Kalman's rank condition (1.6). Then, system (2.4)-(2.5) has only the trivial solution $\Phi=0$ in any one of the following cases:

(i) operator $\gamma$ is global, namely,

$$
\gamma \phi=0 \Longrightarrow \phi=0
$$


(ii) matrix A satisfies the $\epsilon$-closing condition (2.7) with $\epsilon>0$ small enough, and $L$ satisfies the c-gap condition (2.8) and the over-determined scalar problem

$$
\beta^{2} \phi=L \phi \quad \text { and } \quad \gamma \phi=0
$$

has only the trivial solution $\phi \equiv 0$;

(iii) matrix A satisfies the $\epsilon$-closing condition (2.7) with $\epsilon>0$ small enough, and the pair $(L, \gamma)$ is observable.

In order to clarify the main idea, we first make some algebraic preliminaries.

First, since Kalman's rank condition (1.6) is invariant under invertible linear transformation (see [18]), we may assume that $A$ is a diagonal by Jordan blocks. Let $\lambda$ be an eigenvalue of $A$ associated with a Jordan chain of length $r$ :

$$
A E_{k}=\lambda E_{k}+E_{k-1}, \quad 1 \leqslant k \leqslant r \quad \text { with } \quad E_{0}=0 .
$$

For $k=1, \ldots, r$, multiplying system $(2.4)$ by $E_{k}$ and setting $\phi_{k}=\left(E_{k}, \Phi\right)$, we get

$$
L \phi_{k}+\lambda \phi_{k}+\phi_{k-1}=\beta^{2} \phi_{k}, \quad 1 \leqslant k \leqslant r \quad \text { with } \quad \phi_{0}=0 .
$$

Since $L$ is self-adjoint, from the 1 st equation of (2.14)

$$
L \phi_{1}+\lambda \phi_{1}=\beta^{2} \phi_{1}
$$

we deduce that $\lambda$ must be a real number.

Taking the inner product of the 1 st equation of (2.14) with $\phi_{2}$ and that of the 2 nd one with $\phi_{1}$, respectively, we get

$$
\left(L \phi_{1}, \phi_{2}\right)_{H}+\lambda\left(\phi_{1}, \phi_{2}\right)_{H}=\beta^{2}\left(\phi_{1}, \phi_{2}\right)_{H}
$$

and

$$
\left(L \phi_{2}, \phi_{1}\right)_{H}+\lambda\left(\phi_{2}, \phi_{1}\right)_{H}+\left(\phi_{1}, \phi_{1}\right)_{H}=\beta^{2}\left(\phi_{2}, \phi_{1}\right)_{H}
$$

Since $L$ is self-adjoint and $\lambda$ is a real number, it follows from (2.15) and (2.16) that $\phi_{1}=0$.

Repeating the same procedure for 2 nd and $3 r d$ equations of (2.14), we get $\phi_{2}=0$ and so on, until the sub-system (2.14) is reduced to a single equation

$$
\beta^{2} \phi_{r}-L \phi_{r}-\lambda \phi_{r}=0
$$

Without loss of generality, $A$ can be written as

$$
A=\operatorname{diag}(\overbrace{\lambda_{1}, \cdots, \lambda_{1}}^{\sigma_{1}}, \cdots, \overbrace{\lambda_{m}, \cdots, \lambda_{m}}^{\sigma_{m}}),
$$

where $\lambda_{l}$ is an eigenvalue of $A$ with multiplicity $\sigma_{l}(l=1, \cdots, m)$.

Accordingly, defining

$$
\mu_{r}=\mu_{r-1}+\sigma_{r}, \quad r=1, \cdots, m \quad \text { with } \quad \mu_{0}=0,
$$


we regroup the components of $\Phi$ into

$$
\left(\phi^{(1)}, \cdots, \phi^{\left(\mu_{1}\right)}\right),\left(\phi^{\left(\mu_{1}+1\right)}, \cdots, \phi^{\left(\mu_{2}\right)}\right), \cdots \cdots,\left(\phi^{\left(\mu_{m-1}+1\right)}, \cdots, \phi^{\left(\mu_{m}\right)}\right) .
$$

On the other hand, if we replace $A$ by $A+b I$, and $\beta^{2}$ by $\beta^{2}+b$ for any given $b>0$ in (2.4), this will not modify anything in Theorem 2.6. So, without loss of generality, we may assume that $\lambda_{l}>0$ for $1 \leqslant l \leqslant m$.

Denote by $\epsilon_{i}(i=1, \cdots, N)$ the canonical basis vectors in $\mathbb{R}^{N}$ and by $d_{i}$ the $i$ th column-vector of the matrix $D^{T}$. Noting that $D^{T} \epsilon_{i}=d_{i}$ and the subspace $\operatorname{Span}\left\{\epsilon_{\mu_{l-1}+1}, \cdots, \epsilon_{\mu_{l}}\right\}$ is invariant for $A$, by Proposition 2.1, Kalman's rank condition (1.6) implies that $\operatorname{Span}\left\{\epsilon_{\mu_{l-1}+1}, \cdots, \epsilon_{\mu_{l}}\right\} \cap \operatorname{Ker}\left(D^{T}\right)=\{0\}$, namely,

$$
\sum_{i=\mu_{l-1}+1}^{\mu_{l}} \alpha_{i} d_{i}=0 \text { if and only if } \alpha_{i}=0 \text { for } i=\mu_{l-1}+1, \cdots, \mu_{l} \text {. }
$$

Therefore, for any given $l$ with $1 \leqslant l \leqslant m$, the vectors $d_{\mu_{l-1}+1}, \cdots, d_{\mu_{l}}$ are linearly independent.

Now we proceed the proof of Theorem 2.6.

Case (i) From (1.2) and (2.11), we have

$$
D^{T} \mathcal{G} \Phi=\mathcal{G}\left(D^{T} \Phi\right)=0 \Longrightarrow D^{T} \Phi \equiv 0
$$

Then, applying $D^{T}$ to (2.4), it follows that

$$
D^{T} A \Phi \equiv 0
$$

namely,

$$
\sum_{l=1}^{m} \sum_{i=\mu_{l-1}+1}^{\mu_{l}} \lambda_{l} \phi^{(i)} d_{i}=0
$$

We write (2.4) as

$$
L \phi^{(i)}=\left(\beta^{2}+\lambda_{l}\right) \phi^{(i)}, \quad \mu_{l-1}+1 \leqslant i \leqslant \mu_{l}, \quad 1 \leqslant l \leqslant m .
$$

Since $L$ is self-adjoint, the eigen-spaces

$$
\operatorname{Span}\left\{\phi^{\left(\mu_{l-1}+1\right)}, \cdots, \phi^{\left(\mu_{l}\right)}\right\}, \quad 1 \leqslant l \leqslant m
$$

are mutually orthogonal, then we have

$$
\sum_{i=\mu_{l-1}+1}^{\mu_{l}} \lambda_{l} \phi^{(i)} d_{i}=0, \quad 1 \leqslant l \leqslant m
$$

Noting $\lambda_{l}>0$ and the linear independence of $d_{\mu_{l-1}+1}, \cdots, d_{\mu_{l}}$, it follows that

$$
\phi^{(i)}=0, \quad \mu_{l-1}+1 \leqslant i \leqslant \mu_{l}, \quad 1 \leqslant l \leqslant m,
$$

namely, $\Phi \equiv 0$. 
Case (ii) Assume that there exist $l$ and $k$ with $l \neq k$, such that $\phi^{(i)} \neq 0$ for $\mu_{l-1}+1 \leqslant i \leqslant \mu_{l}$, and $\phi^{\left(i^{\prime}\right)} \neq 0$ for $\mu_{k-1}+1 \leqslant i^{\prime} \leqslant \mu_{k}$. There exist $\alpha_{n}$ and $\alpha_{n^{\prime}}$ such that

$$
\beta^{2}+\lambda_{l}=\alpha_{n} \quad \text { and } \quad \beta^{2}+\lambda_{k}=\alpha_{n^{\prime}}
$$

then

$$
\lambda_{l}-\lambda_{k}=\alpha_{n}-\alpha_{n^{\prime}} .
$$

However, because of the $\epsilon$-closing condition (2.7) and the $c$-gap condition (2.8), the above equality cannot be satisfied for $\epsilon>0$ small enough. Therefore, there exists at most an integer $k$ with $1 \leqslant k \leqslant m$, such that

$$
\phi^{(i)}=0, \quad \mu_{l-1}+1 \leqslant i \leqslant \mu_{l}, \quad l \neq k .
$$

Then, (2.5) becomes

$$
D^{T} \mathcal{G} \Phi=\sum_{l=1}^{m} \sum_{i=\mu_{l-1}+1}^{\mu_{l}} \gamma \phi^{(i)} d_{i}=\sum_{i=\mu_{k-1}+1}^{\mu_{k}} \gamma \phi^{(i)} d_{i}=0 .
$$

Noting (2.17) and the linear independence of $d_{\mu_{k-1}+1}, \cdots, d_{\mu_{k}}$, it follows that

$$
L \phi^{(i)}=\left(\beta^{2}+\lambda_{k}\right) \phi^{(i)} \quad \text { with } \quad \gamma \phi^{(i)}=0 \quad \text { for } \quad \mu_{k-1}+1 \leqslant i \leqslant \mu_{k} .
$$

Then the uniqueness of solution to the scalar problem (2.12) implies that

$$
\phi^{(i)}=0, \quad \mu_{k-1}+1 \leqslant i \leqslant \mu_{k},
$$

which, combining with (2.18), leads to

$$
\phi^{(i)}=0, \quad \mu_{l-1}+1 \leqslant i \leqslant \mu_{l}, \quad 1 \leqslant l \leqslant m,
$$

namely, $\Phi \equiv 0$.

Case (iii) Applying $D^{T}$ to (2.4) and noting $W=D^{T} \Phi$, we get

$$
\left(\beta^{2}-a\right) W-\mathcal{L} W=D^{T} A \Phi-a W .
$$

On the other hand, noting the diagonal form of $\mathcal{G}$ in (1.2), condition (2.5) leads to

$$
\mathcal{G} W=\mathcal{G} D^{T} \Phi=D^{T} \mathcal{G} \Phi=0 .
$$

For $1 \leqslant i \leqslant N, 1 \leqslant j \leqslant M$, setting

$$
W=\left(w_{j}\right), \quad D^{T} A \Phi-a W=\left(f_{j}\right) \quad \text { and } \quad D=\left(d_{i j}\right),
$$

we have

$$
w_{j}=\sum_{i=1} d_{j i} \phi^{(i)}=\sum_{l=1}^{m} \sum_{i=\mu_{l-1}+1}^{\mu_{l}} d_{j i} \phi^{(i)}
$$


and

$$
f_{j}=\sum_{l=1}^{m}\left(\lambda_{l}-a\right) \sum_{i=\mu_{l-1}+1}^{\mu_{l}} d_{j i} \phi^{(i)}
$$

Then, taking the $j$ th component of (2.20) and (2.21), we get

$$
\left(\beta^{2}-a\right) w_{j}-L w_{j}=f_{j}
$$

associated with the $D$-observation

$$
\gamma w_{j}=0 .
$$

If $\beta^{2}-a \leqslant 0$, multiplying $(2.22)$ by $w_{j}$, we get

$$
-\left(\beta^{2}-a\right)\left\|w_{j}\right\|_{H}^{2}+\left\|w_{j}\right\|_{V}^{2}=-\left(f_{j}, w_{j}\right)_{H} \leqslant\left\|f_{j}\right\|_{H}\left\|w_{j}\right\|_{H} .
$$

It follows that

$$
c\left\|w_{j}\right\|_{H} \leqslant\left\|f_{j}\right\|_{H}
$$

If $\beta^{2}-a>0$, then $w_{j}$ satisfies the scalar problem (2.9). Since $(L, \gamma)$ is observable, we get again (2.24).

On the other hand, noting that $L$ is self-adjoint, we have

$$
\left(\phi^{(i)}, \phi^{\left(i^{\prime}\right)}\right)_{H}=0, \quad \mu_{l-1}+1 \leqslant i \leqslant \mu_{l}, \quad \mu_{k-1}+1 \leqslant i^{\prime} \leqslant \mu_{k}, \quad l \neq k .
$$

Then it follows from (2.22) that

$$
\left\|f_{j}\right\|_{H}^{2} \leqslant \sup _{1 \leqslant l \leqslant m}\left|a-\lambda_{l}\right|^{2} \sum_{l=1}^{m}\left\|\sum_{i=\mu_{l-1}+1}^{\mu_{l}} d_{j i} \phi^{(i)}\right\|_{H}^{2}=\sup _{1 \leqslant l \leqslant m}\left|a-\lambda_{l}\right|^{2}\left\|w_{j}\right\|_{H}^{2},
$$

hence, by the $\epsilon$-closing condition (2.7) we get

$$
\left\|f_{j}\right\|_{H} \leqslant \sup _{1 \leqslant l \leqslant m}\left|a-\lambda_{l}\right|\left\|w_{j}\right\|_{H} \leqslant \epsilon\left\|w_{j}\right\|_{H}
$$

Thus, it follows from (2.24) that

$$
\sum_{i=\mu_{l-1}+1}^{\mu_{l}} d_{i j} \phi^{(i)}=0, \quad 1 \leqslant j \leqslant M, \quad 1 \leqslant l \leqslant m,
$$

provided that $\epsilon<c$, namely, we have

$$
\sum_{i=\mu_{l-1}+1}^{\mu_{l}} d_{i} \phi^{(i)}=0, \quad 1 \leqslant l \leqslant m
$$


Then, the linear independence of $d_{\mu_{l-1}+1}, \ldots, d_{\mu_{l}}$ implies that

$$
\phi^{(i)}=0, \quad \mu_{l-1}+1 \leqslant i \leqslant \mu_{l}, \quad 1 \leqslant l \leqslant m .
$$

namely, $\Phi \equiv 0$. The proof is then complete.

Theorem 2.6 shows that a seemingly difficult uniqueness of solution to a complex system can be established through the observability of an over-determined scalar problem. This idea will be developed for studying the asymptotic stability and the asymptotic synchronization of second order evolution systems.

The first case (i) corresponds to the globally distributed observation. In this case, similarly to the finite dimension case, without any additional assumptions on the matrix $A$ either on the operator $L$, only the Kalman's rank condition is sufficient for the uniqueness of solution to the over-determined system. In the second case (ii), the condition $\gamma \phi=0$ is not a priori assumed to yield $\phi=0$. However, thanks to the $c$-gap condition (2.8), the uniqueness of solution to the scalar problem (2.12), which is weaker than the observability inequality, implies also the uniqueness of solution to the over-determined system. In the last case (iii), the observability inequality (2.9) does not come from the well-posedness, but rather from Carleman's estimation, and requires additional information on the differential operator $L$ as well as on the geometric condition of the domain (see Sect. 5 for wave equations).

\section{Asymptotic stability}

Recall that the operators $L$ and $\gamma$ satisfy conditions (2.1)-(2.3). In what follows, we assume furthermore that the operator $\gamma$ is compact from $V$ into $V^{\prime}$.

Let $A$ and $D$ be symmetric and positive semi-definite matrices. Consider the following second order evolution equations:

$$
U^{\prime \prime}+\mathcal{L} U+A U+D \mathcal{G} U^{\prime}=0
$$

Defining the linear operator $\mathcal{A}$ by

$$
\mathcal{A}(U, \widehat{U})=(\widehat{U},-\mathcal{L} U-A U-D \mathcal{G} \widehat{U})
$$

with the domain

$$
D(\mathcal{A})=\{(U, \widehat{U}) \in \mathcal{V} \times \mathcal{V}: \quad \mathcal{L} U+A U+D \mathcal{G} \widehat{U} \in \mathcal{V}\}
$$

we transform (3.1) into an abstract formulation as follows:

$$
(U, \widehat{U})^{\prime}=\mathcal{A}(U, \widehat{U})
$$

Proposition 3.1. The operator $\mathcal{A}$ defined in (3.2)-(3.3) generates a semi-group of contractions in the space $\mathcal{V} \times \mathcal{H}$. Moreover, $\mathcal{A}^{-1}$ is compact in $\mathcal{V} \times \mathcal{H}$.

Proof. Using (2.1)-(2.3), we first check that $\mathcal{A}$ is dissipative:

$$
(\mathcal{A}(U, \widehat{U}),(U, \widehat{U}))_{\mathcal{V} \times \mathcal{H}}=-\langle\mathcal{D} \mathcal{G} \widehat{U}, \widehat{U}\rangle_{\mathcal{V}^{\prime}, \mathcal{V}} \leqslant 0 .
$$

We next show that $R(\mathcal{A})=\mathcal{V} \times \mathcal{H}$. Then by Hill-Yosida's Theorem (see [26]), it generates a semi-group of contractions in the space $\mathcal{V} \times \mathcal{H}$. 
To this end, for any given $(F, \widehat{F}) \in \mathcal{V} \times \mathcal{H}$, we solve the equation $\mathcal{A}(U, \widehat{U})=(F, \widehat{F})$, namely,

$$
\widehat{U}=F, \quad \mathcal{L} U+A U=-(D \mathcal{G} F+\widehat{F}) .
$$

Noting (2.1) and the convention of pivot space, it follows that

$$
(U, \Phi)_{\mathcal{V}}+(A U, \Phi)_{\mathcal{H}}=-\langle D \mathcal{G} F+\widehat{F}, \Phi\rangle_{\mathcal{V}^{\prime}, \mathcal{V}}, \quad \forall \Phi \in \mathcal{V}
$$

which, due to Lax-Milgram's Lemma, admits a unique solution $U \in \mathcal{V}$ with the continuous dependence:

$$
\|U\|_{\mathcal{V}} \leq c\|(D \mathcal{G} F+\widehat{F})\|_{\mathcal{V}^{\prime}}
$$

Since the operator $\gamma$ is compact from $V$ to $V^{\prime}$, so is the operator $\mathcal{G}$ from $\mathcal{V}$ to $\mathcal{V}^{\prime}$. On the other hand, noting that the embeddings $V \subset H \subset V^{\prime}$ are compact, so the map

$$
(F, \widehat{F}) \rightarrow U=-(\mathcal{L}+A)^{-1}(D \mathcal{G} F+\widehat{F})
$$

is compact from $\mathcal{V} \times \mathcal{H}$ to $\mathcal{V}$. Therefore the map

$$
(F, \widehat{F}) \rightarrow(U, \widehat{U})=\left(-(\mathcal{L}+A)^{-1}(D \mathcal{G} F+\widehat{F}), F\right)
$$

is compact from $\mathcal{V} \times \mathcal{H}$ into $\mathcal{V} \times \mathcal{H}$. Therefore, $\mathcal{A}^{-1}$ is compact in $\mathcal{V} \times \mathcal{H}$.

Definition 3.2. System (3.1) is asymptotically (strongly) stable in $\mathcal{V} \times \mathcal{H}$ if for any given initial data $\left(U_{0}, U_{1}\right) \in$ $\mathcal{V} \times \mathcal{H}$, the corresponding solution $U$ satisfies

$$
\left(U(t), U^{\prime}(t)\right) \rightarrow(0,0) \quad \text { in } \mathcal{V} \times \mathcal{H} \text { as } t \rightarrow+\infty
$$

Proposition 3.3. Assume that system (3.1) is asymptotically stable in $\mathcal{V} \times \mathcal{H}$. Then we necessarily have Kalman's rank condition (1.6).

Proof. If (1.6) fails, then there exist $E \in \mathbb{R}^{N}$ and $\lambda \in \mathbb{R}$, such that

$$
A E=\lambda E \quad \text { and } \quad D E=0 .
$$

Applying $E$ to (3.1) and setting $\phi=(E, U)$, we get a conservative equation:

$$
\phi^{\prime \prime}+L \phi+\lambda \phi=0,
$$

which is never asymptotically stable.

The following theorem suggests to handle the asymptotic stability of the whole system (3.1) by means of the uniqueness of a scalar problem.

Theorem 3.4. Let the pair of matrices $(A, D)$ satisfy Kalman's rank condition (1.6). Then, system (3.1) is asymptotically stable in any one of the situations described in Theorem 2.6.

Proof. Noting that $\mathcal{A}^{-1}$ is compact in the space $\mathcal{V} \times \mathcal{H}$, by the classic theory of semi-groups (see [2, 3]), the dissipative system (3.1) is asymptotically stable if and only if $\mathcal{A}$ has no pure imaginary eigenvalues.

Now, let $\beta \in \mathbb{R}$ and $(\Phi, \Psi) \in \mathcal{V} \times \mathcal{H}$, such that

$$
\mathcal{A}(\Phi, \Psi)=i \beta(\Phi, \Psi)
$$


It follows that

$$
\mathcal{L} \Phi+A \Phi+i \beta D \mathcal{G} \Phi=\beta^{2} \Phi .
$$

Since $\mathcal{L}+A$ is coercive, we have $\beta \neq 0$. Then, noting that $\mathcal{L}$ and $D \mathcal{G}$ are symmetric and positive semi-definite, we deduce that (3.6) is equivalent to system (2.4)-(2.5), which has only the trivial solution $\Phi \equiv 0$ by virtue of Theorem 2.6.

Thanks to Theorem 2.6, the above theorem confirms well the asymptotic stability of a large class of linear dissipative systems (see examples in Sect. 5).

On the other hand, due to the absence of a general uniqueness theorem, although the equivalence between the controllability and the $D$-observability for wave systems has been established in $[16,17,19]$, however, except few specific systems, we don't know if a system of wave equations is actually controllable or not.

\section{Asymptotic SYNCHRONizATion By GRoups}

By Proposition 3.3, when the pair of matrices $(A, D)$ does not satisfy Kalman's rank condition (1.6), the dissipative system (3.1) is not asymptotically stable. Instead of asymptotic stability, we have introduced the asymptotic synchronization by $p$-groups in Definition 1.2.

Now let $S_{r}$ be the full row-rank matrix of order $\left(n_{r}-n_{r-1}-1\right) \times\left(n_{r}-n_{r-1}\right)$ :

$$
S_{r}=\left(\begin{array}{ccccc}
1 & -1 & & & \\
& 1 & -1 & & \\
& & \ddots & \ddots & \\
& & & 1 & -1
\end{array}\right), \quad 1 \leqslant r \leqslant p .
$$

Define the $(N-p) \times N$ matrix $C_{p}$ of synchronization by $p$-groups as

$$
C_{p}=\left(\begin{array}{llll}
S_{1} & & & \\
& S_{2} & & \\
& & \ddots & \\
& & & S_{p}
\end{array}\right) .
$$

Let $\epsilon_{1}, \ldots, \epsilon_{N}$ be the vectors of the canonical basis of $\mathbb{R}^{N}$. Defining

$$
e_{r}=\sum_{i=n_{r-1}+1}^{n_{r}} \epsilon_{i}, \quad 1 \leqslant r \leqslant p
$$

we have

$$
\operatorname{Ker}\left(C_{p}\right)=\operatorname{Span}\left\{e_{1}, \ldots, e_{p}\right\}
$$

Then the asymptotic synchronization by $p$-groups (1.12) can be equivalently written as

$$
C_{p}\left(U(t), U^{\prime}(t)\right) \rightarrow(0,0) \quad \text { in }(V \times H)^{N-p} \text { as } t \rightarrow+\infty .
$$

Before starting the study on the asymptotic synchronization by $p$-groups, we first give some algebraic preliminaries. 
Definition 4.1. Matrix $A$ satisfies the condition of $C_{p}$-compatibility if

$$
A K \operatorname{Ker}\left(C_{p}\right) \subseteq \operatorname{Ker}\left(C_{p}\right) .
$$

Proposition 4.2. The condition of $C_{p}$-compatibility (4.6) is equivalent to the existence of a symmetric and positive semi-definite matrix $\bar{A}_{p}$ of order $(N-p)$, such that

$$
\left(C_{p} C_{p}^{T}\right)^{-1 / 2} C_{p} A=\bar{A}_{p}\left(C_{p} C_{p}^{T}\right)^{-1 / 2} C_{p}
$$

Proof. It is sufficient to note that $\operatorname{Ker}\left(\left(C_{p} C_{p}^{T}\right)^{-1 / 2} C_{p}\right)=\operatorname{Ker}\left(C_{p}\right)$. Then, we conclude by virtue of Lemma 3.3 in [23].

Moreover, the reduced matrix $\bar{A}_{p}$ can be explicitly given by

$$
\bar{A}_{p}=\left(C_{p} C_{p}^{T}\right)^{-1 / 2} C_{p} A C_{p}^{T}\left(C_{p} C_{p}^{T}\right)^{-1 / 2},
$$

which shows that the reduced matrix $\bar{A}_{p}$ is also symmetric and positive semi-definite.

Definition 4.3. Matrix $D$ satisfies the condition of strong $C_{p}$-compatibility if

$$
\operatorname{Ker}\left(C_{p}\right) \subseteq \operatorname{Ker}(D) .
$$

Proposition 4.4. The condition of strong $C_{p}$-compatibility (4.9) is equivalent to the existence of a symmetric and positive semi-definite matrix $R$ of order $(N-p)$, such that

$$
D=C_{p}^{T} R C_{p} .
$$

Proof. Noting that $\operatorname{Ker}(\sqrt{D})=\operatorname{Ker}(D)$, it follows from (4.9) that

$$
\operatorname{Im}(\sqrt{D})=(\operatorname{Ker}(\sqrt{D}))^{\perp}=(\operatorname{Ker}(D))^{\perp} \subseteq \operatorname{Im}\left(C_{p}^{T}\right) .
$$

Thus, there exists a matrix $\widehat{R}$ of order $(N-p) \times N$, such that $\sqrt{D}=C_{p}^{T} \widehat{R}$, which gives the expression in (4.10) with $R=\widehat{R} \widehat{R}^{T}$.

Moreover, setting

$$
\bar{D}_{p}=\left(C_{p} C_{p}^{T}\right)^{1 / 2} R\left(C_{p} C_{p}^{T}\right)^{1 / 2}
$$

we easily check that $\bar{D}_{p}$ satisfies

$$
\left(C_{p} C_{p}^{T}\right)^{-1 / 2} C_{p} D=\bar{D}_{p}\left(C_{p} C_{p}^{T}\right)^{-1 / 2} C_{p}
$$

This justifies well the terminology "the condition of strong $C_{p}$-compatibility".

Remark 4.5. Noting the special form of (4.4), the conditions of compatibility (4.6) and (4.9) are equivalent to the row-sum conditions by blocks (1.15).

Proposition 4.6. Assume that $A$ satisfies the condition of $C_{p}$-compatibility (4.7) and $D$ is given by (4.10). Then the asymptotic synchronization by p-groups of system (3.1) is equivalent to the asymptotic stability of the following reduced system with the variable $W=\left(w^{(1)}, \ldots, w^{(N-p)}\right)^{T}$ :

$$
W^{\prime \prime}+\mathcal{L} W+\bar{A}_{p} W+\bar{D}_{p} G W^{\prime}=0 .
$$


Proof. Applying the matrix $\left(C_{p} C_{p}^{T}\right)^{-1 / 2} C_{p}$ to system (3.1), noting the conditions of $C_{p^{-}}$-compatibility (4.7) and (4.12) and setting

$$
W=\left(C_{p} C_{p}^{T}\right)^{-1 / 2} C_{p} U
$$

we get the self-closed reduced system (4.13). Moreover, since the reduced matrices $\bar{A}_{p}$ and $\bar{D}_{p}$ are still symmetric and positive semi-definite, the asymptotic stability of the reduced system (4.13) can be obtained by applying Theorem 3.4.

The necessity of the conditions of $C_{p}$-compatibility is a delicate question. Intuitively, we think that this question is intrinsically linked with the rank of Kalman's matrix. In what follows, we will give a lower bound on the rank of Kalman's matrix in Theorem 4.7. Next, in Theorem 4.8, we show that the necessity of the conditions of $C_{p}$-compatibility is a consequence of the minimality of the rank of Kalman's matrix. We establish the asymptotic synchronization by $p$-groups in Theorem 4.10. We clarify the two kinds of asymptotic synchronizations by $p$-groups in Theorem 4.11 at the end of the section.

Theorem 4.7. Assume that system (3.1) is asymptotically synchronizable by p-groups. Then we necessarily have

$$
\operatorname{rank}\left(D, A D, \ldots, A^{N-1} D\right) \geqslant N-p
$$

Proof. Assume that

$$
\operatorname{rank}\left(D, A D, \ldots, A^{N-1} D\right)=N-q \quad \text { with } q>p
$$

Noting that $A$ is symmetric, by Proposition 2.1, without loss of generality, we may assume that there exists a subspace $\operatorname{Span}\left\{E_{1}, \ldots, E_{q}\right\}$ such that

$$
A E_{r}=\lambda_{r} E_{r} \quad \text { and } \quad D E_{r}=0, \quad r=1, \ldots, q .
$$

Since

$$
\operatorname{dim} \operatorname{Im}\left(C_{p}^{T}\right)+\operatorname{dim} \operatorname{Span}\left\{E_{1}, \ldots, E_{q}\right\}=N-p+q>N
$$

there exists a unit vector $E \in \operatorname{Span}\left\{E_{1}, \ldots, E_{q}\right\} \cap \operatorname{Im}\left(C_{p}^{T}\right)$. Let $x \in \mathbb{R}^{N-p}$ such that $E=C_{p}^{T} x$. Setting $\phi=$ $(E, U)=\left(x, C_{p} U\right)$, by $(4.5)$ we have

$$
\left(\phi, \phi^{\prime}\right) \rightarrow(0,0) \quad \text { in } V \times H \quad \text { as } t \rightarrow+\infty .
$$

On the other hand, since $E \in \operatorname{Span}\left\{E_{1}, \ldots, E_{q}\right\}$, we can write $E=\sum_{r=1}^{q} a_{r} E_{r}$. Then, setting $\phi_{r}=\left(E_{r}, U\right)$ for $r=1, \ldots, q$, we get

$$
\phi=\sum_{r=1}^{q} a_{r}\left(E_{r}, U\right)=\sum_{r=1}^{q} a_{r} \phi_{r} .
$$

Applying $E_{r}$ to system (3.1), we get

$$
\phi_{r}^{\prime \prime}+L \phi_{r}+\lambda_{r} \phi_{r}=0, \quad r=1, \ldots, q
$$


associated with the initial data:

$$
t=0: \quad \phi_{r}=v_{r}, \quad \phi_{r}^{\prime}=0, \quad r=1, \ldots, q,
$$

where the functions $v_{r}$ are given by

$$
L v_{r}=\beta_{r}^{2} v_{r}, \quad r=1, \ldots, q .
$$

Since the eigenfunctions $v_{1}, \ldots, v_{q}$ are orthonormal, so are the solutions $\phi_{1}, \ldots, \phi_{q}$. Then we have

$$
\|\phi\|_{H}^{2}=\left\|\sum_{r=1}^{q} a_{r} \phi_{r}\right\|_{H}^{2}=\sum_{r=1}^{q}\left|a_{r}\right|^{2}\left\|\phi_{r}\right\|_{H}^{2}=\sum_{r=1}^{q}\left|a_{r}\right|^{2} \equiv 1 .
$$

This contradicts the convergence (4.16). The proof is then complete.

Now we show that the necessity of the conditions of $C_{p}$-compatibility is in fact the consequence of the minimality of the rank of Kalman's matrix.

Theorem 4.8. Assume that system (3.1) is asymptotically synchronizable by p-groups under the minimal rank condition

$$
\operatorname{rank}\left(D, A D, \ldots, A^{N-1} D\right)=N-p
$$

Then, $A$ satisfies the condition of $C_{p}$-compatibility (4.7) and $D$ satisfies the condition of strong $C_{p}$-compatibility (4.10).

Proof. By Proposition 2.1, the rank condition (4.20) implies the existence of $p$ orthonormal vectors $E_{1}, \ldots, E_{p}$ such that

$$
A E_{r}=\lambda_{r} E_{r} \quad \text { and } \quad D E_{r}=0, \quad r=1, \ldots, p .
$$

Let $\beta_{r}^{2}$ be the eigenvalue of $L$ associated with the eigenvector $v_{r}$, defined by (4.19). Setting

$$
\beta_{r}^{2}=\mu_{r}^{2}+\lambda_{r}>0
$$

we check easily that for each $r$ with $1 \leqslant r \leqslant p$, the function $\mathrm{e}^{i \beta_{r} t} v_{r} E_{r}$ is a solution to system (3.1). Then, by (4.5) we have

$$
\mathrm{e}^{i \beta_{r} t} v_{r} C_{p} E_{r} \rightarrow 0 \text { in } V^{N-p} \text { as } t \rightarrow+\infty
$$

It follows thus that

$$
C_{p} E_{r}=0, \quad r=1, \ldots, p,
$$

namely,

$$
\operatorname{Ker}\left(C_{p}\right)=\operatorname{Span}\left\{E_{1}, \ldots, E_{p}\right\},
$$

which together with (4.21) imply conditions (4.6) and (4.9). 
Proposition 4.9. Assume that $A$ satisfies the condition of $C_{p}$-compatibility (4.7) and $D$ is given by (4.10). Then,

$$
\operatorname{rank}\left(C_{p}\left(D, A D, \ldots, A^{N-1} D\right)\right)=\operatorname{rank}\left(D, A D, \ldots, A^{N-1} D\right) .
$$

Proof. By the condition of $C_{p^{-}}$-compatibility (4.6) and the condition of strong $C_{p^{-}}$-compatibility (4.9), we have

$$
\operatorname{Ker}\left(C_{p}\right) \subseteq \operatorname{Ker}\left(\begin{array}{c}
D \\
D A \\
\vdots \\
D A^{N-1}
\end{array}\right)
$$

namely,

$$
\operatorname{Im}\left(D, A D, \ldots, A^{N-1} D\right) \subseteq \operatorname{Im}\left(C_{p}^{T}\right)
$$

Then it follows that

$$
\operatorname{Ker}\left(C_{p}\right) \cap \operatorname{Im}\left(D, A D, \ldots, A^{N-1} D\right) \subseteq \operatorname{Ker}\left(C_{p}\right) \cap \operatorname{Im}\left(C_{p}^{T}\right)=\{0\},
$$

which is equivalent to (4.22).

As for the asymptotic stability, the following theorem indicates that the asymptotic synchronization by $p$-groups of system (3.1) can be reduced to the uniqueness of solution to an over-determined scalar problem.

Theorem 4.10. Let the pair $(A, D)$ satisfy Kalman's rank condition (4.20). Assume that $A$ satisfies the condition of $C_{p}$-compatibility (4.7) and $D$ is given by (4.10). Then, system (3.1) is asymptotically synchronizable by p-groups in any one of the cases described in Theorem 2.6.

Proof. By Proposition 4.6, it suffices to show the asymptotic stability of the reduced system (4.13). By Theorem 3.4 , system (4.13) is asymptotically stable under the condition

$$
\operatorname{rank}\left(\bar{D}_{p}, \bar{A}_{p} \bar{D}_{p}, \ldots, \bar{A}_{p}^{N-p-1} \bar{D}_{p}\right)=N-p .
$$

In order to complete the proof, we will show that the rank condition (4.20) is equivalent to (4.23).

In fact, noting (4.7) and (4.12), we have

$$
\left(\bar{D}_{p}, \bar{A}_{p} \bar{D}_{p}, \ldots, \bar{A}_{p}^{N-p-1} \bar{D}_{p}\right)=\left(C_{p} C_{p}^{T}\right)^{-\frac{1}{2}} C_{p}\left(D, A D, \ldots, A^{N-1} D\right),
$$

then it follows that

$$
\operatorname{rank}\left(\bar{D}_{p}, \bar{A}_{p} \bar{D}_{p}, \ldots, \bar{A}_{p}^{N-p-1} \bar{D}_{p}\right)=\operatorname{rank}\left(C_{p}\left(D, A D, \ldots, A^{N-1} D\right)\right) .
$$

By Proposition 4.9, we obtain (4.23).

Theorem 4.11. Assume that $A$ satisfies the condition of $C_{p}$-compatibility (4.7) and $D$ is given by (4.10). If system (3.1) is asymptotically synchronizable by p-groups, then for any given initial data $\left(U_{0}, U_{1}\right) \in \mathcal{V} \times \mathcal{H}$, there exist some scalar functions $u_{1}, \ldots, u_{p}$ such that

$$
\left(u^{(k)}(t)-u_{r}(t),\left(u^{(k)}\right)^{\prime}(t)-u_{r}^{\prime}(t)\right) \rightarrow(0,0) \quad \text { in } V \times H
$$

as $t \rightarrow+\infty$ for all $n_{r-1}+1 \leqslant k \leqslant n_{r}$ and $1 \leqslant r \leqslant p$. 
Proof. Noting (4.4), there exist some real numbers $\alpha_{r s}$ with $\alpha_{r s}=\alpha_{s r}$, such that

$$
A e_{r}=\sum_{s=1}^{p} \alpha_{r s} e_{s}, \quad r=1, \ldots, p .
$$

Then, applying $e_{r}$ to $(3.1)$ and setting $v_{r}=\left(e_{r}, U\right)$ for $r=1, \ldots p$, we get

$$
v_{r}^{\prime \prime}+L v_{r}+\sum_{s=1}^{p} \alpha_{r s} v_{s}=0
$$

associated with the initial data

$$
t=0: \quad v_{r}=\left(U_{0}, e_{r}\right), \quad v_{r}^{\prime}=\left(U_{1}, e_{r}\right)
$$

Setting

$$
S=\left(\begin{array}{c}
e_{1}^{T} \\
\vdots \\
e_{p}^{T} \\
C_{p}
\end{array}\right)
$$

by (4.5) we have

$$
S U=\left(\begin{array}{c}
\left(e_{1}, U\right) \\
\vdots \\
\left(e_{p}, U\right) \\
C_{p} U
\end{array}\right) \rightarrow\left(\begin{array}{c}
v_{1} \\
\vdots \\
v_{p} \\
0
\end{array}\right) \quad \text { in } \mathcal{V} \times \mathcal{H} \text { as } t \rightarrow+\infty
$$

On the other hand, since

$$
S e_{r}=\left\|e_{r}\right\|^{2} \epsilon_{r}, \quad r=1, \ldots, p
$$

setting $u_{r}=v_{r} /\left\|e_{r}\right\|^{2}$, it follows that

$$
U \rightarrow S^{-1}\left(\begin{array}{c}
v_{1} \\
\vdots \\
v_{p} \\
0
\end{array}\right)=\sum_{r=1}^{p} v_{r} S^{-1} \epsilon_{r}=\sum_{r=1}^{p} \frac{v_{r}}{\left\|e_{r}\right\|^{2}} e_{r}=\sum_{r=1}^{p} u_{r} e_{r}
$$

in $\mathcal{V} \times \mathcal{H}$ as $t \rightarrow+\infty$. The proof is thus complete.

Remark 4.12. The convergence in (1.12) will be called the asymptotic synchronization by $p$-groups in the consensus sense, and the convergence in (4.24) will be called in the pinning sense. Theorem 4.11 clarifies that the two notions are simply the same. However, the functions $u_{1}, \ldots, u_{p}$ given by Theorem 4.11 are not unique. In fact, any functions $\widehat{u}_{1}, \ldots, \widehat{u}_{p}$ such that for $r=1, \ldots, p$,

$$
\left(\widehat{u}_{r}-u_{r}, \widehat{u}_{r}^{\prime}-u_{r}^{\prime}\right) \rightarrow(0,0) \quad \text { in } \mathcal{V} \times \mathcal{H} \quad \text { as } t \rightarrow+\infty
$$

satisfy also (4.24). 


\section{Applications}

\subsection{Wave equations with boundary damping}

We will give some classical examples to illustrate possible applications of the developed abstract theory. However, the approach is quite flexible and can be easily applied to other types of wave equations with memory and variable density [15], outside a star-shaped domain [28] and orbital stability [30].

Let $\Omega$ be a bounded domain in $\mathbb{R}^{n}$ with the boundary $\Gamma=\Gamma_{1} \cup \Gamma_{0}$ such that mes $\left(\Gamma_{1}\right)>0$. Consider the following system of wave equations:

$$
\begin{cases}U^{\prime \prime}-\Delta U+A U=0 & \text { in } \mathbb{R}^{+} \times \Omega, \\ U=0 & \text { on } \mathbb{R}^{+} \times \Gamma_{0}, \\ \partial_{\nu} U+D U^{\prime}=0 & \text { on } \mathbb{R}^{+} \times \Gamma_{1},\end{cases}
$$

where $\partial_{\nu}$ denotes the outward normal derivative on the boundary.

Multiplying system (5.1) by a test function $\Phi \in\left(H_{\Gamma_{0}}^{1}(\Omega)\right)^{N}$ and integrating by parts, we get the following variational formulation:

$$
\int_{\Omega}\left(U^{\prime \prime}, \Phi\right) \mathrm{d} x+\int_{\Omega}(\nabla U, \nabla \Phi) \mathrm{d} x+\int_{\Omega}(A U, \Phi) \mathrm{d} x+\int_{\Gamma_{1}}\left(D U^{\prime}, \Phi\right) \mathrm{d} \Gamma=0 .
$$

Let $L$ and $\gamma$ be defined by

$$
\langle L u, \phi\rangle=\int_{\Omega} \nabla u \cdot \nabla \phi \mathrm{d} x \quad \text { and } \quad\langle\gamma v, \phi\rangle=\int_{\Gamma_{1}} v \phi \mathrm{d} \Gamma
$$

respectively. Setting $\mathcal{L}$ and $\mathcal{G}$ as in (1.2), the variational equation (5.2) can be rewritten as

$$
U^{\prime \prime}+\mathcal{L} U+A U+D \mathcal{G} U^{\prime}=0
$$

Obviously, the operators $L$ and $\gamma$ defined by (5.3) satisfy conditions (2.1)-(2.3). Then by Proposition 3.1, system (5.4) generates a semi-group of contractions.

\subsubsection{Case with Gap Condition}

We first consider a specific situation of (5.1) on a rectangular domain

$$
\Omega=(0, \pi) \times(0, a \pi), \quad \Gamma_{0}=\{(0, y) \cup(\pi, y), \quad 0<y<a \pi\},
$$

where $a>0$ is a parameter.

Theorem 5.1. Let $a^{2}$ be a rational. Assume that the pair $(A, D)$ satisfies the rank condition (1.6). Then system (5.1) is asymptotically stable in $\left(H_{\Gamma_{0}}^{1}(\Omega) \times L^{2}(\Omega)\right)^{N}$, provided that A satisfies the $\epsilon$-closing condition with $\epsilon>0$ small enough.

Proof. Consider the following eigen-problem:

$$
\begin{cases}-\Delta \phi=\alpha \phi & \text { in } \Omega \\ \phi=0 & \text { on } \Gamma_{0} \\ \partial_{\nu} \phi=0 & \text { on } \Gamma_{1}\end{cases}
$$


with the additional observation

$$
\phi=0, \quad \text { on } \Gamma_{1} \text {. }
$$

A straightforward computation gives the eigenvalues and the associated eigenvectors as follows:

$$
\alpha_{k, l}=k^{2}+\frac{l^{2}}{a^{2}}, \quad \phi_{k, l}=\sin (k x) \cos \left(\frac{l y}{a}\right) .
$$

We check easily, or directly by Carleman's unique continuation theorem [5, 10], that the over-determined system (5.6)-(5.7) has only the trivial solution.

Moreover, when $a^{2}$ is a rational, the eigenvalues satisfy the $c$-gap condition:

$$
\left|\alpha_{k, l}-\alpha_{p, q}\right|=\left|k^{2}-p^{2}+\frac{l^{2}-q^{2}}{a^{2}}\right| \geqslant c>0 .
$$

Then by Theorem 3.4, system (5.1) is asymptotically stable, provided that $A$ satisfies the $\epsilon$-closing condition with $\epsilon>0$ small enough.

\subsubsection{Case with Observability Inequality}

In this section, we assume that there exists $x_{0} \in R^{n}$, such that setting $m=x-x_{0}$, we have $(m \cdot \nu) \leqslant 0$ on $\Gamma_{0}$, where $\nu$ denotes the outward normal vector on the boundary.

Proposition 5.2. The pair $(L, \gamma)$ defined by (5.3) is observable.

Proof. By definition, we have to show that

$$
\int_{\Omega}|\phi|^{2} \mathrm{~d} x \leqslant c \int_{\Omega}|f|^{2} \mathrm{~d} x
$$

for any given solution $\phi$ to the following over-determined scalar problem:

$$
\begin{cases}\beta^{2} \phi+\Delta \phi=f & \text { in } \Omega, \\ \phi=0 & \text { on } \Gamma_{0} \\ \partial_{\nu} \phi=0 & \text { on } \Gamma_{1}\end{cases}
$$

with the observation

$$
\phi=0 \quad \text { on } \Gamma_{1} .
$$

Let us first recall Rellich's identity

$$
2 \int_{\Omega} \Delta \phi(m \cdot \nabla \phi) \mathrm{d} x=(n-2) \int_{\Omega}|\nabla \phi|^{2} \mathrm{~d} x+\int_{\Gamma}\left(2 \partial_{\nu} \phi(m \cdot \nabla \phi)-(m \cdot \nu)|\nabla \phi|^{2}\right) \mathrm{d} \Gamma
$$

for all $\phi \in H^{2}(\Omega)$, where $m=x-x_{0}$.

Then, multiplying the equation in $(5.10)$ by $2 m \cdot \nabla \phi+(n-1) \phi$ and integrating by parts, we have

$$
\int_{\Omega}\left(|\beta \phi|^{2}+|\nabla \phi|^{2}\right) \mathrm{d} x-\int_{\Gamma_{0}}(m \cdot \nu)\left|\partial_{\nu} \phi\right|^{2} \mathrm{~d} \Gamma=-\int_{\Omega} f(2 m \cdot \nabla \phi+(n-1) \phi) \mathrm{d} x .
$$


Since $(m \cdot \nu) \leqslant 0$ on $\Gamma_{0}$, we get a stronger version than $(5.9)$ :

$$
\int_{\Omega}\left(|\beta \phi|^{2}+|\nabla \phi|^{2}\right) \mathrm{d} x \leqslant c \int_{\Omega}|f|^{2} \mathrm{~d} x
$$

The proof is complete.

Theorem 5.3. Assume that the pair $(A, D)$ satisfies the rank condition (4.20). Let $A$ satisfy the condition of $C_{p^{-}}$ compatibility (4.7) and $D$ be given by (4.10). Assume furthermore that A satisfies the $\epsilon$-closing condition (2.7) with $\epsilon>0$ small enough. Then system (5.1) is asymptotically synchronizable by p-groups in $\left(H_{\Gamma_{0}}^{1}(\Omega) \times L^{2}(\Omega)\right)^{N}$.

Proof. Noting that the trace operator $\gamma$ is compact from $H_{\Gamma_{0}}^{1}(\Omega)$ into $L^{2}\left(\Gamma_{1}\right)$. Furthermore, by Proposition 5.2, the pair $(L, \gamma)$ is observable. Then, by Theorem 4.10 we get the conclusion.

Remark 5.4. The inequality (5.12) is much stronger than (5.9). It can be served to establish the decay rate, for example, the uniform decay rate as in [22]:

$$
\left\|C_{p}\left(U(t), U^{\prime}(t)\right)\right\|_{(\mathcal{V} \times \mathcal{H})^{N-p}} \leqslant M \mathrm{e}^{-\omega t}\left\|C_{p}\left(U_{0}, U_{1}\right)\right\|_{(\mathcal{V} \times \mathcal{H})^{N-p}}
$$

where $M$ and $\omega$ are positive constants, or the polynomial decay rate for the smooth initial data:

$$
\left\|C_{p}\left(U(t), U^{\prime}(t)\right)\right\|_{(\mathcal{V} \times \mathcal{H})^{N-p}}=O\left(t^{-\delta}\right),
$$

where the constant $\delta>0$ is independent of the initial data. We refer to $[7,27]$ and the references therein for the recent progress on the polynomial stability of indirectly damped wave equations.

\subsection{Wave equations with distributed damping}

Let $\Omega$ be a bounded domain in $\mathbb{R}^{n}$ with smooth boundary $\Gamma$. Consider the following coupled system of wave equations:

$$
\begin{cases}U^{\prime \prime}-\Delta U+A U+D U^{\prime}=0 & \text { in } \mathbb{R}^{+} \times \Omega \\ U=0 & \text { on } \mathbb{R}^{+} \times \Gamma\end{cases}
$$

Multiplying system (5.15) by a test function $\Phi \in\left(H_{0}^{1}(\Omega)\right)^{N}$ and integrating by parts, we get the following variational formulation:

$$
\int_{\Omega}\left(U^{\prime \prime}, \Phi\right) \mathrm{d} x+\int_{\Omega}(\nabla U, \nabla \Phi) \mathrm{d} x+\int_{\Omega}(A U, \Phi) \mathrm{d} x+\int_{\omega}\left(D U^{\prime}, \Phi\right) \mathrm{d} \Gamma=0 .
$$

Let $L$ and $\gamma$ be defined by

$$
\langle L u, \phi\rangle=\int_{\Omega} \nabla u \cdot \nabla \phi \mathrm{d} x \quad \text { and } \quad\langle\gamma v, \phi\rangle=\int_{\Omega} v \phi \mathrm{d} x
$$

respectively. The variational problem (5.16) can be rewritten as

$$
U^{\prime \prime}+\mathcal{L} U+A U+D \mathcal{G} U^{\prime}=0
$$

Theorem 5.5. Assume that the pair $(A, D)$ satisfies the rank condition (4.20). Assume furthermore that $A$ satisfies the condition of $C_{p}$-compatibility (4.7) and $D$ is given by (4.10). Then system (5.15) is asymptotically synchronizable by p-groups in $\left(H_{0}^{1}(\Omega) \times L^{2}(\Omega)\right)^{N}$. 
Proof. It is a direct consequence of Theorem 4.10 .

\subsection{Kirchhoff plate equations with shear force damping}

In this section, $\Omega$ is a star-shaped domain in $\mathbb{R}^{2}$, occupied by an elastic thin plate. Let $\mu$ with $0<\mu<1 / 2$ be the Poisson ratio. Let the shear force operator $B_{1}$ and the bending moment operator $B_{2}$ be defined by

$$
\left\{\begin{array}{l}
B_{1} \phi=2 \nu_{1} \nu_{2} \partial_{x y} \phi-\nu_{1}^{2} \partial_{y y} \phi-\nu_{2}^{2} \partial_{x x} \phi \\
B_{2} \phi=\left(\nu_{1}^{2}-\nu_{2}^{2}\right) \partial_{x y} \phi+\nu_{1} \nu_{2}\left(\partial_{x x} \phi-\partial_{y y} \phi\right) .
\end{array}\right.
$$

Consider the following system (see $[11,12]$ for more precise description):

$$
\begin{cases}U^{\prime \prime}+\Delta^{2} U+A U=0 & \text { in } \mathbb{R}^{+} \times \Omega, \\ \Delta U+(1-\mu) B_{1} U=0 & \text { on } \mathbb{R}^{+} \times \Gamma, \\ \partial_{\nu} \Delta U+(1-\mu) \partial_{\tau} B_{2} U=D U^{\prime} & \text { on } \mathbb{R}^{+} \times \Gamma,\end{cases}
$$

where $\partial_{\tau}$ denotes the tangential derivative on the boundary. For the sake of simplicity, the shear force damping is acted on the whole boundary $\Gamma$.

Define the symmetric bilinear form by

$$
a(\phi, \psi)=\phi_{x x} \psi_{x x}+\phi_{y y} \psi_{y y}+\mu\left(\phi_{x x} \psi_{y y}+\phi_{y y} \psi_{x x}\right)+2(1-\mu) \phi_{x y} \psi_{x y} .
$$

Recall the Green's formula (see (4.3.20) in [11]):

$$
\begin{aligned}
\int_{\Omega} \Delta^{2} \phi \psi \mathrm{d} x \mathrm{~d} y= & \int_{\Omega} a(\phi, \psi) \mathrm{d} x \mathrm{~d} y \\
& +\int_{\Gamma}\left(\partial_{\nu} \Delta \phi+(1-\mu) \partial_{\tau} B_{2} \phi\right) \psi \mathrm{d} \Gamma-\int_{\Gamma}\left(\Delta \phi+(1-\mu) B_{1} \phi\right) \partial_{\nu} \psi \mathrm{d} \Gamma .
\end{aligned}
$$

Multiplying system (5.20) by a test function $\Phi \in\left(H^{2}(\Omega)\right)^{N}$ and integrating by parts, we get the following variational formulation:

$$
\int_{\Omega}\left(U^{\prime \prime}, \Phi\right) \mathrm{d} x \mathrm{~d} y+\int_{\Omega} a(U, \Phi) \mathrm{d} x \mathrm{~d} y+\int_{\Omega}(A U, \Phi) \mathrm{d} x \mathrm{~d} y+\int_{\Gamma}\left(D U^{\prime}, \Phi\right) \mathrm{d} \Gamma=0
$$

Let $L$ and $\gamma$ be defined by

$$
\langle L u, \phi\rangle=\int_{\Omega} a(u, \phi) \mathrm{d} x \mathrm{~d} y \quad \text { and } \quad\langle\gamma v, \phi\rangle=\int_{\Gamma} v \phi \mathrm{d} \Gamma .
$$

(5.23) can be interpreted as

$$
U^{\prime \prime}+\mathcal{L} U+A U+D \mathcal{G} U^{\prime}=0
$$

which, by Proposition 3.1, is well-posed in the sense of semi-group of contractions in the space $\left(H^{2}(\Omega) \times L^{2}(\Omega)\right)^{N}$.

Proposition 5.6. The pair $(L, \gamma)$ defined by (5.24) is observable.

Proof. We have to show

$$
\int_{\Omega}|\phi|^{2} \mathrm{~d} x \mathrm{~d} y \leqslant c \int_{\Omega}|f|^{2} \mathrm{~d} x \mathrm{~d} y
$$


for any given solution $\phi$ to the following over-determined scalar problem:

$$
\begin{cases}\beta^{2} \phi-\Delta^{2} \phi=f & \text { in } \Omega \\ \Delta \phi+(1-\mu) B_{1} \phi=0 & \text { on } \Gamma \\ \partial_{\nu} \Delta \phi+(1-\mu) \partial_{\tau} B_{2} \phi=0 & \text { on } \Gamma\end{cases}
$$

with the observation

$$
\phi=0 \quad \text { on } \Gamma .
$$

Recall the following identity (see Lem. 4.5.1 in [11]):

$$
\begin{aligned}
\int_{\Omega} \Delta^{2} \phi(m \cdot \nabla \phi) \mathrm{d} x \mathrm{~d} y= & \int_{\Omega} a(\phi, \phi) \mathrm{d} x \mathrm{~d} y+\frac{1}{2} \int_{\Gamma}(m \cdot \nu) a(\phi, \phi) \mathrm{d} \Gamma \\
& +\int_{\Gamma}\left(\partial_{\nu} \Delta \phi+(1-\mu) \partial_{\tau} B_{2} \phi\right)(m \cdot \nabla \phi) \mathrm{d} \Gamma-\int_{\Gamma}\left(\Delta \phi+(1-\mu) B_{1} \phi\right) \partial_{\nu}(m \cdot \nabla \phi) \mathrm{d} \Gamma .
\end{aligned}
$$

Multiplying the equation in (5.27) by $m \cdot \nabla \phi$ and integrating by parts, we have

$$
\int_{\Omega}|\beta \phi|^{2} \mathrm{~d} x \mathrm{~d} y+\int_{\Omega} a(\phi, \phi) \mathrm{d} x \mathrm{~d} y+\frac{1}{2} \int_{\Gamma}(m \cdot \nu) a(\phi, \phi) \mathrm{d} \Gamma=-\int_{\Omega} f(m \cdot \nabla \phi) \mathrm{d} x \mathrm{~d} y,
$$

which implies (5.26). The proof is complete.

Theorem 5.7. Assume that the pair $(A, D)$ satisfies the rank condition (4.20). Let $A$ satisfy the condition of $C_{p^{-}}$ compatibility (4.7), and $D$ be given by (4.10). Assume furthermore that A satisfies the $\epsilon$-closing condition (2.7) with $\epsilon>0$ small enough. Then system (5.20) is asymptotically synchronizable by p-groups in $\left(H^{2}(\Omega) \times L^{2}(\Omega)\right)^{N}$.

Proof. Since the trace operator $\gamma$ is compact from $H^{2}(\Omega)$ into $L^{2}(\Gamma)$, and the pair $(L, \gamma)$ is observable, then, applying Theorem 4.10 we get the conclusion.

\section{Comments}

In this section, we want to mention several possibilities to enlarge the research field on this topic.

\subsection{Kirchhoff plate with distributed damping}

Let $A, D_{1}, D_{2}$ be symmetric and semi-positive matrices. Consider the following system

$$
\begin{cases}U^{\prime \prime}+\Delta^{2} U+A U+D_{1} U^{\prime}-D_{2} \Delta U^{\prime}=0 & \text { in } \mathbb{R}^{+} \times \Omega \\ U=\partial_{\nu} U=0 & \text { on } \mathbb{R}^{+} \times \Gamma\end{cases}
$$

We will investigate the asymptotic behavior of system (6.1) under the joint action of the damping given by the shear force $D_{1} U^{\prime}$ and the bending moment $D_{2} \Delta U^{\prime}$.

Theorem 6.1. System (6.1) is asymptotically stable in $\left(H_{0}^{2}(\Omega) \times L^{2}(\Omega)\right)^{N}$ if and only if $(A, D)$ satisfies Kalman's rank condition (1.6) with $D=\left(D_{1}, D_{2}\right)$.

Proof. The asymptotic stability of system (6.1) is equivalent to the uniqueness of solution to the eigen-problem

$$
\begin{cases}\Delta^{2} \Phi+A \Phi=\beta^{2} \Phi & \text { in } \Omega \\ \Phi=\partial_{\nu} \Phi=0 & \text { on } \Gamma\end{cases}
$$


associated with the mixed observations

$$
D_{1} \Phi=0 \quad \text { and } \quad D_{2} \Delta \Phi=0 \quad \text { in } \Omega .
$$

Obviously, Kalman's rank condition (1.6) with $D=\left(D_{1}, D_{2}\right)$ is still necessary for the uniqueness of solution to (6.2)-6.3). We then examine the sufficiency. Applying $D_{1}$ to (6.2), it follows that

$$
\Delta^{2} D_{1} \Phi+D_{1} A \Phi=\beta^{2} D_{1} \Phi \text { in } \Omega
$$

The first observation in (6.3) implies that $D_{1} A \Phi=0$ in $\Omega$. A simple recurrence successively gives

$$
D_{1} \Phi=D_{1} A \Phi=\cdots=D_{1} A^{N-1} \Phi=0 .
$$

On the other hand, the second observation in (6.3) implies that $D_{2} \Phi=0$ in $\Omega$. Then, by the same procedure as for $D_{1}$, we get

$$
D_{2} \Phi=D_{2} A \Phi=\cdots=D_{2} A^{N-1} \Phi=0
$$

By (6.5) and (6.6), it follows that

$$
\Phi \in \operatorname{Ker}\left(\begin{array}{c}
D^{T} \\
D^{T} A \\
\vdots \\
D^{T} A^{N-1}
\end{array}\right)=\left\{\operatorname{Im}\left(D, A D, \ldots, A^{N-1} D\right)\right\}^{\perp}=\{0\},
$$

then $\Phi \equiv 0$. The proof is complete.

Remark 6.2. In this example, the feedbacks are global. This case is easy to be treated, but it gives the idea of generalizing Theorem 2.6 to the case of several observations. Once it is realized, we can consider the problems with mixed damping, in particular, plate equations with distributed and boundary damping, wave equations with memory and thermal source, etc. We are waiting for a wide field of applications and discover new challenge in the forthcoming works.

\subsection{System of elasticity}

Let $u=\left(u_{1}, u_{2}, u_{3}\right)^{T}$ denote the displacement of an elastic body which occupies a bounded domain $\Omega \subset \mathbb{R}^{3}$ with smooth boundary $\Gamma$.

Define

$$
\epsilon_{i j}(u)=\frac{1}{2}\left(\frac{\partial u_{i}}{\partial x_{j}}+\frac{\partial u_{j}}{\partial x_{i}}\right) \text { and } \quad \sigma_{i j}(u)=\lambda \sum_{k=1}^{3} \epsilon_{k k}(u) \delta_{i j}+2 E \epsilon_{i j}(u),
$$

where $\lambda>0$ is Lamé's coefficient and $E>0$ Young's module. The evolution of the elastic body obeys the following system:

$$
\begin{cases}u^{\prime \prime}-\operatorname{div}(\sigma(u))+A u=0 & \text { in } \mathbb{R}^{+} \times \Omega \\ \sigma(u) \nu=D u^{\prime} & \text { on } \mathbb{R}^{+} \times \Gamma\end{cases}
$$

where $\sigma(u)=\left(\sigma_{i j}(u)\right)$, and $\nu$ denotes the outside normal vector on the boundary $\Gamma$. 
Let

$$
u^{(l)}=\left(\begin{array}{c}
u_{1}^{(l)} \\
u_{2}^{(l)} \\
u_{3}^{(l)}
\end{array}\right), \quad U=\left(\begin{array}{c}
u^{(1)} \\
\vdots \\
u^{(N)}
\end{array}\right), \quad \Sigma(U)=\left(\begin{array}{c}
\sigma\left(u^{(1)}\right) \\
\vdots \\
\sigma\left(u^{(N)}\right)
\end{array}\right)
$$

and

$$
\operatorname{Div}(\Sigma(U))=\left(\begin{array}{ccc}
\operatorname{div}\left(\sigma\left(u^{(1)}\right)\right) & \cdots & 0 \\
\vdots & \ddots & \vdots \\
\vdots & \ddots & \vdots \\
0 & \cdots & \operatorname{div}\left(\sigma\left(u^{(N)}\right)\right)
\end{array}\right)
$$

Then, the motion of $N$ elastic bodies is governed by the following evolution equations

$$
\begin{cases}U^{\prime \prime}-\operatorname{Div}(\Sigma(U))+\mathcal{A} U=0 & \text { in } \mathbb{R}^{+} \times \Omega \\ \Sigma(U) \nu=\mathcal{D} U^{\prime} & \text { on } \mathbb{R}^{+} \times \Gamma\end{cases}
$$

where $\mathcal{A}$ and $\mathcal{D}$ are some matrices of order $3 N$ with real constant coefficients.

Let us examine a specific situation in which

$$
\mathcal{A}=\left(a_{i j} I_{3}\right)_{1 \leqslant i, j \leqslant N} \quad \text { and } \quad \mathcal{D}=\left(d_{i j} I_{3}\right)_{1 \leqslant i, j \leqslant N} .
$$

Let

$$
A=\left(a_{i j}\right)_{1 \leqslant i, j \leqslant N} \quad \text { and } \quad D=\left(a_{i j}\right)_{1 \leqslant i, j \leqslant N} .
$$

Clearly,

$$
\operatorname{rank}\left(\mathcal{D}, \mathcal{A D}, \ldots, \mathcal{A}^{3 N-1} D\right)=3 N
$$

holds if and only if

$$
\operatorname{rank}\left(D, A D, \ldots, A^{N-1} D\right)=N
$$

If (6.12) fails, then there exist $\lambda \in \mathbb{R}$ and $x=\left(x_{i}\right) \in \mathbb{R}^{N}$, such that

$$
D x=0 \quad \text { and } \quad A x=\lambda x .
$$

Setting

$$
\mathcal{E}=\left(\begin{array}{c}
x_{1} I_{3} \\
\vdots \\
x_{N} I_{3}
\end{array}\right)
$$

we easily check that

$$
\mathcal{A E}=\lambda \mathcal{E} \quad \text { and } \quad \mathcal{D E}=0 .
$$


Then, applying $\mathcal{E}$ to system (6.8) and setting

$$
\phi=\sum_{l=1} x_{l} u^{(l)}
$$

we find that $\phi$ satisfies the following system:

$$
\begin{cases}\phi^{\prime \prime}-\operatorname{div}(\sigma(\phi))+\lambda \phi=0 & \text { in } \mathbb{R}^{+} \times \Omega \\ \sigma(\phi) \nu=0 & \text { on } \mathbb{R}^{+} \times \Gamma\end{cases}
$$

which is conservative, therefore never asymptotically stable.

Thus, the rank condition (6.11) is indeed necessary for the stability. However, if the matrices $\mathcal{A}$ and $\mathcal{D}$ are not of the form (6.9), the stability of system (6.8) is a largely open problem.

Of course, the above discussion can also be carried out for many other situations, such as Timoshenko beam $[1,9]$, Bresse beam [25], thermo-elastic plate [4, 6], viscoelastic Petrovsky-type equation [14, 24] and Maxwell equations [8].

Acknowledgements. This work was partially supported by National Natural Science Foundation of China under Grant 11831011.

\section{REFERENCES}

[1] F. Ammar-Khodja, A. Benabdallah, J.E. Munoz Rivera and R. Racke, Energy decay for Timoshenko systems of memory type. J. Diff. Eqs. 194 (2003) 82-115.

[2] W. Arendt and C.J. Batty, Tauberian theorems and stability of one-parameter semi-groups. Trans. Amer. Math. Soc. 306 (1988) 83-852.

[3] C.D. Benchimol, A note on weak stabilization of contraction semi-groups. SIAM J. Control Optim. 16 (1978) 373-379.

[4] L. de Teresa and E. Zuazua, Controllability of the linear system of thermoelastic plates. Adv. Diff. Eqs. 1 (1996) 369-402.

[5] N. Garofalo and F. Lin, Uniqueness of solution for elliptic operators. A geometric-variational approach. Commun. Pure Appl. Math. 40 (1987) 347-366.

[6] S.W. Hansen and B. Zhang, Boundary control of a linear thermo-elastic beam. J. Math. Anal. Appl. 210 (1997) $182-205$.

[7] J. Hao, B. Rao, Influence of the hidden regularity on the stability of partially damped systems of wave equations. J. Math. Pure Appl. 143 (2020) 257-286.

[8] B.V. Kapitonov, Stabilization and exact boundary controllability for Maxwell's equations. SIAM J. Control Optim. 32 (1994) 408-420.

[9] J.U. Kim and Y. Renardy, Boundary control of the Timoshenko beam. SIAM J. Control Optim. 25 (1987) 1417-1429.

[10] H. Koch and D. Tataru, Carleman estimates and uniqueness of solution for second-order elliptic equations with nonsmooth coefficients. Commun. Pure Appl. Math. 54 (2001) 339-360.

[11] J.E. Lagnese, Boundary Stabilization of Thin Plates. SIAM, Study in applied mathematics. Philadelphia (1989).

[12] J.E. Lagnese and J.-L. Lions, Modelling, Analysis and Control of Thin Plates, Recherches en Mathématiques Appliquées, Masson, Paris (1988).

[13] I. Lasiecka and R. Triggiani, Uniform stabilization of a shallow shell model with nonlinear boundary dampings. J. Math. Anal. Appl. 269 (2002) 642-688.

[14] F. Li and G. Du, General energy decay for a degenerate viscoelastic Petrovsky-type plate equation with boundary feedback. J. Appl. Anal. Comp. 8 (2018) 390-401.

[15] F. Li and Z. Jia, Global existence and stability of a class of nonlinear evolution equations with hereditary memory and variable density Bound. Value Probl. 37 (2019) 23.

[16] T.-T. Li and B. Rao, Criteria of Kalman's type to the approximate controllability and the approximate synchronization for a coupled system of wave equations with Dirichlet boundary controls. SIAM J. Control Optim. 54 (2016) 49-72.

[17] T.-T. Li and B. Rao, On the approximate boundary synchronization for a coupled system of wave equations: Direct and indirect controls. ESAIM: COCV 24 (2018) 1675-1704.

[18] T.T. Li and B. Rao, Kalman's criterion on the uniqueness of continuation for the nilpotent system of wave equations. $C$. $R$. Acad. Sci. Paris, Ser. I 356 (2018) 1188-1194.

[19] T.-T. Li and B. Rao, On the approximate boundary synchronization for a coupled system of wave equations: Direct and indirect boundary controls. ESAIM: COCV 24 (2018) 1675-1704.

[20] T.-T. Li and B. Rao, Boundary Synchronization for Hyperbolic Systems. Progress in Non Linear Differential Equations and Their Applications, Subseries in Control, Vol. 94. Birkhaüser (2019). 
[21] T.-T. Li and B. Rao, Uniqueness theorem for partially observed elliptic systems and application to asymptotic synchronization. C. R. Math. 358 (2020) 285-295.

[22] T.-T. Li and B. Rao, Uniform synchronization of second order evolution equations. In preparation (2020).

[23] T.-T. Li, B. Rao and Y.M. Wei, Generalized exact boundary synchronization for second order evolution systems. Disc. Contin. Dyn. Syst. 34 (2014) 2893-2905.

[24] F. Li, Sh. Xi, K. Xu and X. Xue, Dynamic properties for nonlinear viscoelastic Kirchhoff-type equation with acoustic control boundary conditions II. J. Appl. Anal. Comput. 9 (2019) 2318-2332.

[25] Z. Liu and B. Rao, Energy decay rate of the thermoelastic Bresse system. Z. Angew. Math. Phys. 60 (2009) 54-69.

[26] A. Pazy, Semi-groups of Linear Operators and Applications to Partial Differential Equations. Applied Mathematical Sciences. Springer-Verlag (1983).

[27] B. Rao, On the sensitivity of the transmission of boundary dissipation for strongly coupled and indirectly damped systems of wave equations. Z. Angew. Math. Phys. 70 (2019) 25.

[28] L. Ren and J. Xin, Almost global existence for the Neumann problem of quasilinear wave equations outside star-shaped domains in 3D. Electr. J. Diff. Eqs. 312 (2018) 1-22.

[29] F. Trèves, Basic Linear Partial Differential Equations. Pure and Applied Mathematics, Vol. 62. Academic Press, New York/London (1975).

[30] X. Zheng, J. Xin and X. Peng, Orbital stability of periodic traveling wave solutions to the generalized long-short wave equations. J. Appl. Anal. Comput. 9 (2019) 2389-2408. 